\title{
Multi-functioning home bot with integrated feedback system
}

\author{
Lalit Mohan Satapathy*, Samir Kumar Bastia, Nihar Mohanty \\ Department of Electrical and Electronics Engineering, \\ Siksha ' $\mathrm{O}$ ' Anusandhan, Deemed to be University, 751030 Bhubaneswar, India \\ *Corresponding author, e-mail: alitmohan.satapathy@gmail.com
}

\begin{abstract}
Now-a-days, automation in home is becoming more popular. It always remains important to consider accuracy and speed in a said job. This paper is addressing an Arduino Mega micro-controller based Home Bot, which has several aspects like cleaning of the floor and on-off switches as per requirement, theft detection and monitoring. The ability of the proposed model is auto pathfinding when ever hurdles appear and auto detection when a trouble appears inside the Home Bot. It is verified that the cost of this module is around 21\%, compared to the robots available in the market having features of only floor cleaning.
\end{abstract}

Keywords: arduino mega, automatic vacuum cleaner, home automation, smart feedback system, smart home bot.

Copyright $\mathbb{C} 2019$ APTIKOM - All rights reserved.

\section{Introduction}

In this busy and sophisticated life, there is hardly any time for rest. Different upcoming technologies are claiming to make the daily life activities simpler without compromising its speed. Household activities are one of the inseparable parts of daily life. May it be sweeping the floor, to turning off the electrical appliances, everything needs to be remembered and done at an expected time. Home automation provides a better solution to these kinds of things. These activities are feedback based, through the internet for real-time notification and control which is well-known as the Internet of Things (IoT). The multifunctioning bot proposed in this paper provides a solution to daily life activities. It not only cleans the floor but also automatically turns off the electrical appliances whenever there is absence of human beings. The design is compact in size to reach every corner of the room and consumes low power. As the system is operated with smart feedback, the user can know and fix the problem without help of an expert, which provides a unique feature to this system.

The paper is organized as follows. Section II provides a literature review. In section III, we explained the hard ware design of the prototype. In Section IV the experiments which confirm the validation of the design are established. The development of science and technology is a challenging one in the field of automation. Not only in Industry but also in the medical field and domestic use, automation is used to reduce the complexity of time and burden. The attitude and acceptance towards automation are increasing day by day.

Recently, Gutmann et al [1] have investigated the attitude of customers towards a systematic floor cleaner and how such a robot influences their lifestyle. Their disclosures suggest that people can save their productive time more than one hour per week as well as driving towards more beneficial lives. Efficient cleaning is seen as a basic highlight of the residential environment which supports tremendous affirmation of the robot. Sung et al [2] have investigated the long-term acceptance of robots at home [2]. With their finding as pre-adoption, adoption, adaptation, and retention, they took the primary step toward setting up a system for Household Robot Biology. Dai \& Chen [3] have proposed the grid scanning algorithm for the obstacle detection based on electric map with sensor array. The finding indicates that the detection system improves cleaning and path search ability significantly. Hong et al [4] have prepared a small cleaning robot which includes two different modules for vacuum cleaning and mopping. The robot takes an irregular way for floor cleaning but it had no arrangement for zone covering. Lang \& Chee [5] have proposed the fuzzy controller based mobile robot for floor cleaning, obstacle avoidance and navigation.

As per history "Whirlwind" was the first vacuum cleaner invented by W. McGaffey in the year 1868 [6]. The first automatic floor cleaner robot named Mint was developed in 2010 by Jen Steffen, with Global Positioning System (GPS). Thomas et al [7] have proposed the use of a microprocessor, 
ATMEGA328 and IR sensors to build an automatic floor cleaning robot in his paper "An Advanced mobile Robot for Floor Cleaning". Rashid [8] developed a mobile phone operated Dual Tone MultiFrequency (DTMF) controlled automatic floor cleaner in 2016. Image processing based automatic vacuum cleaner for detection of dust and mapping of the area is proposed by Prashar [9]. This system contains automatic charging feature. The major disadvantage of this device is that it requires a PC for operation. Saunders [10] proposed a method called "Teach Me-Show Me" through which a home robot learns itself with the help of end users. This is completely Personalization of robot which uses real-time programming concept and fuzzy logic to do a task. The advantage is here the client can program the robot for following a few instructions. The disadvantages are bulky in nature, a complex algorithm, expensive and low speed [10]. 'Roomba 700' the first compatible, efficient and reliable automatic vacuum cleaner was launched in the year 2013, with a smart mapping technology and build in camera. It uses a spiral algorithm and zigzag mode to clean the floor and an inbuilt timer to start the cleaning operation automatically at a user specified time [11].

Table 1 shows the comparison between IRobot (Roomba), which is the first model of an automated vacuum cleaner with the latest Samsung home bot and our proposed design. Through detailed study, it is observed that none of the above models are using feedback system and auto path recovery technique. This motivates towards the development of a smart, low cost, multi-functioning home bot which not only cleans the floor efficiently but also keeps in mind the safety of the system.

Table 1. Comparison of Different automated Vacuum Cleaner

\begin{tabular}{ccccc}
\hline SL. No. & Features & Irobot (ROMBAA) & SAMSUNG (VR9000) & Proposed product \\
\hline 1 & RF Module & Yes & Yes & Yes \\
2 & LCD & No & Yes & Yes \\
3 & IR Sensor & No & No & Yes \\
4 & Mode of operation & Random & Spiral, Zig-Zag & Spiral, Zig-Zag \\
5 & Monitoring mode & No & No & Yes \\
6 & Speed control of fan & No & No & Yes \\
7 & Automatic load switching & No & No & Yes \\
8 & Automatic Fault detection & No & Yes \\
\hline
\end{tabular}

\section{Research Method}

The silent features of the proposed bot are.

a. Automatic fault detection when the switch of the bot is on.

b. Cleaning the dust particle present in the floor.

c. Obstacle avoidance during forwarding movement.

d. Original path recovery after the obstacle is detected.

e. Staircase avoidance.

f. Wall detection and avoidance.

g. Emergency stops when anyone tries to lift it up.

h. Automatic human detection for switching off the electrical loads.

i. Night monitoring with the local alert (buzzer).

j. Speed control of fan used in the vacuum cleaner.

To achieve the above requirements, the hardware implementation consists of 5 different parts as (i) self-troubleshooting (ii) vacuum cleaning (iii) Arduino Mega as decision maker (iv) RF module is used to transmit the data wirelessly and (v) Security alert. The implementation was done keeping in view the maximum utilization of all the modules. The different sensors and hardware are connected to the Arduino mega as given in the block diagram Figure 1. The vacuum cleaner motor is used for vacuum cleaning purpose and when the dust collector is removed it works as a fan. 


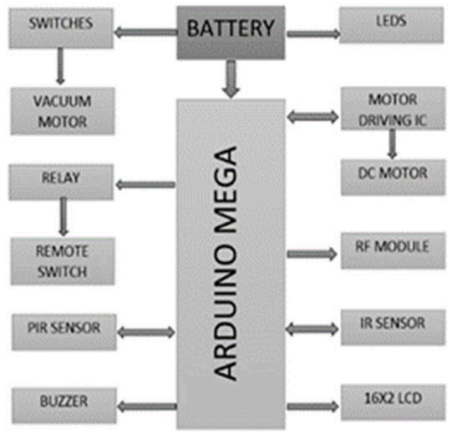

(a)

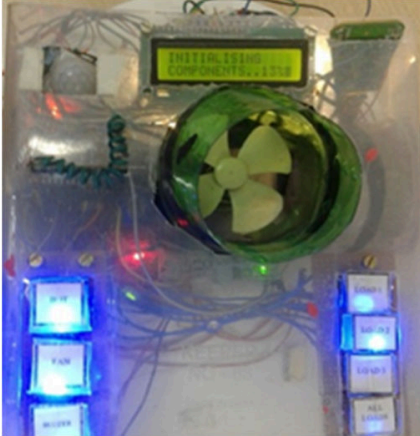

(b)

Figure 1. (a) Block Diagram of the vacuum cleaner, (b) Implemented model

\subsection{Arduino Mega}

The Arduino Mega 2560 is a microcontroller with 54 digital input/output pins and is powered by ATmega2560. Out of 54 input/output pins, 15 can be used as PWM outputs and 16 as analog inputs. It also contains $16 \mathrm{MHz}$ crystal oscillator and $256 \mathrm{~KB}$ flash memory. The Arduino Mega is programmed through the open source Arduino IDE (Matijevic \& Cvjetkovic, 2016) [12].

\subsection{Motor Driving IC (L239D)}

The microcontrollers are operating at a very low voltage and current, so it cannot drive the motor which requires $250 \mathrm{~mA}$. For this purpose, motor driver (ICs) are used. The motordriver is a current amplifier, which takes the low current signal and gives amplified high current signal. The direction of the motor can be controlled by the help of the motor driver. The driver is designed to accept standard TTL logic levels to enable or disable the device independently as per the input signals. The L239D is a high voltage, high current full-bridge driver and capable of driving loads such as DC Motors, stepper motors, and relays for the industrial purpose. The module is powerful enough for supporting DC Motors with voltages between 5 and $35 \mathrm{~V}$, with a maximum current of $2 \mathrm{~A}$. Battery Operated motor has several gears inside it which minimizes the speed and gives a perfect grip to the wheels connected for driving the bot. The motor driving IC is also used as a battery indicator for indicating the availability of power.

\subsection{RF Remote Module}

$\mathrm{RF}$ remote module is operating at $315 \mathrm{MHz}$ for transferring data between Arduino and Load. The receiver Sensitivity is great than $90 \mathrm{dBm}$ and the remote can control the Load within a distance of 100 meters.

\subsection{Infrared Receiver (IR)}

IR sensor (700 $\mathrm{nm}$ to $1400 \mathrm{~nm}$ ) is used for obstacle detection, staircase avoidance, and emergency stop purpose. The IR Sensor transmits an infrared signal, this infrared signal bounces from the obstacle and the signal is received at the infrared receiver. The decision is made based on the signal received. With the help of IR sensor, the functions like emergency stop, a monitoring mode, and the spot cleaning can be activated.

\subsection{Passive Infrared Sensor (PIR)}

PI sensor is used to detect the presence of a human being within a room. The PIR sensor range is up to 10 meters at an angle of +15 o or -15 o.

\subsection{4-Channel Relay}

4-Channel relay is connected to the Arduino for controlling the home appliances in a sequence as (i) fan (ii) light (iii) room-heater and (iv) TV set. Relay triggers the switch which is connected to a high voltage and takes low current and voltage for operation. 4 input pins of the relay are connected to Arduino which takes $5 \mathrm{~V}$ supply from it and can trigger up to $10 \mathrm{~A}, 250 \mathrm{~V}$ supply. 


\section{Results and Discussion}

The hardware connections are implemented as per the Figure 1 and the programming is done with the help of Arduino IDE. The automatic fault is detected when any of the wire is disconnected and any sensor is out of order. When a fault occurs, the resistance value of the system deviates from the defined value range. During the fault the sensor's resistance value are observed through the program as:

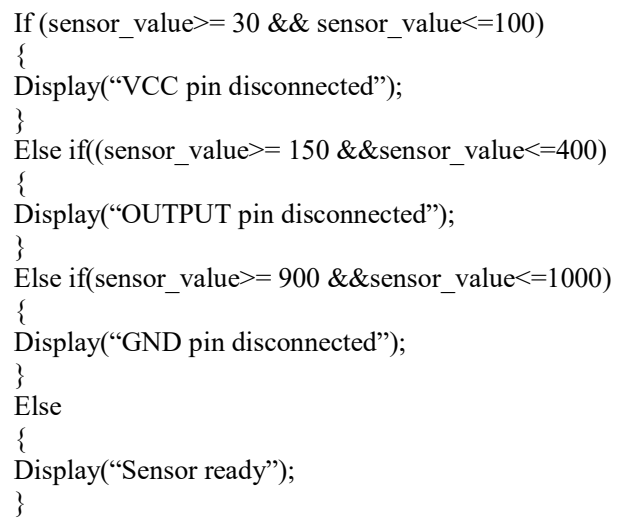

A dc motor is used for creating low pressure and collecting the dust in a dust collector and the filter is used for isolating the fan from the collected dust. Now, whenever the dust collector is removed by remote, it simply acts like a fan. The IR sensing element lining towards left, right and front are used for performing three important jobs as (i) avoiding staircase, (ii) performing an emergency stop, (iii) restrict the area of the room. IR sensor receives the signal unless and until it is being lifted up or the signal is reflected from a black object. Deviation of the signal is programmed for performing the above assigned job. For auto path recovery purpose Arduino Mega is taking a decision based on information's from sensors and different logics.

For auto path recovery, the program logic used as:

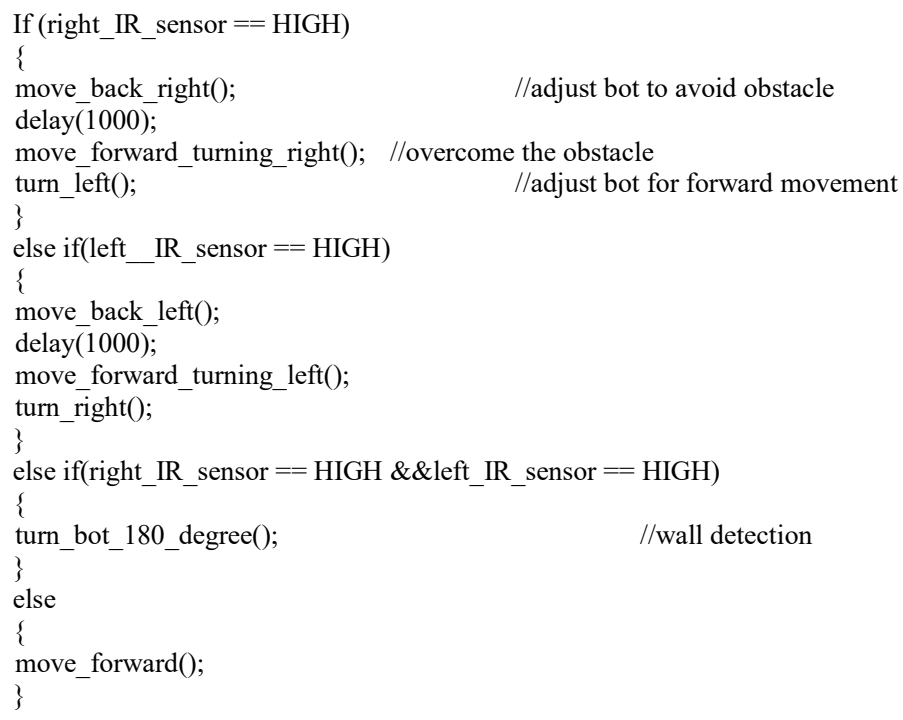

On monitoring mode and load switching condition, the Passive Infrared Sensor checks the human presence. As per the programme with non-availability of human, it updates the system time and switches off the entire load after five minutes.

For human detection, the program logic used is as 


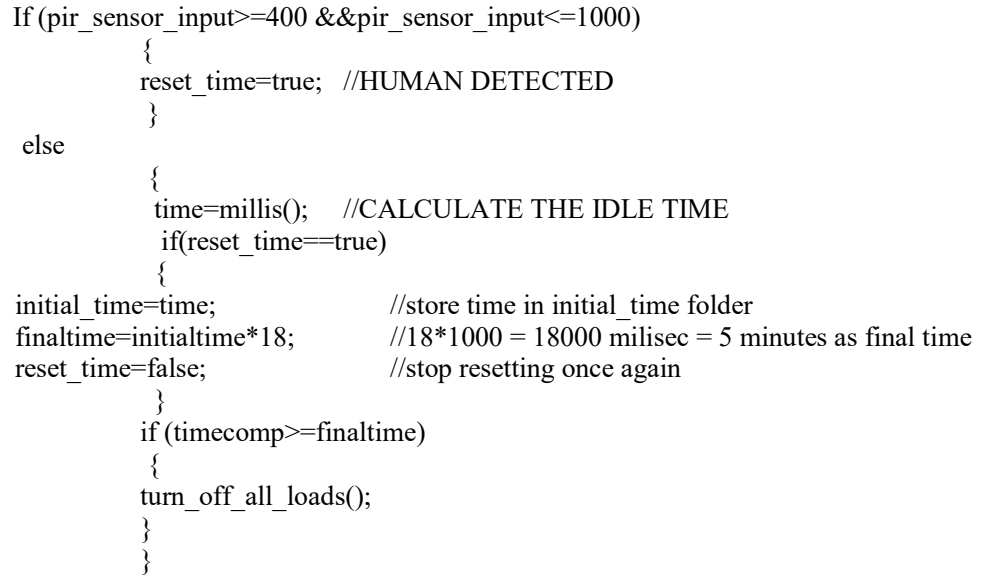

L2389D IC works on the principle of H-bridge. It simply takes power from the battery (12V$30 \mathrm{~V}$ ) and pulse width modulation (PWM) signal from Arduino and steps down the voltage, current (according to the PWM input) and supply it to the DC motor. The direct connection between $12 \mathrm{~V}$ DC battery and Arduino is not possible because Arduino takes a maximum of 5V and 50mA. So, if the input is given to Arduino from L2389D then it can detect the voltage level of the DC battery safely.

In night monitoring mode the buzzer is $\mathrm{ON}$, whenever the system found some random movement around it or change in analog value rapidly, it will turn on the buzzer for local alert. The working of the total system can be better understood from flow chart as shown in Figure 2.

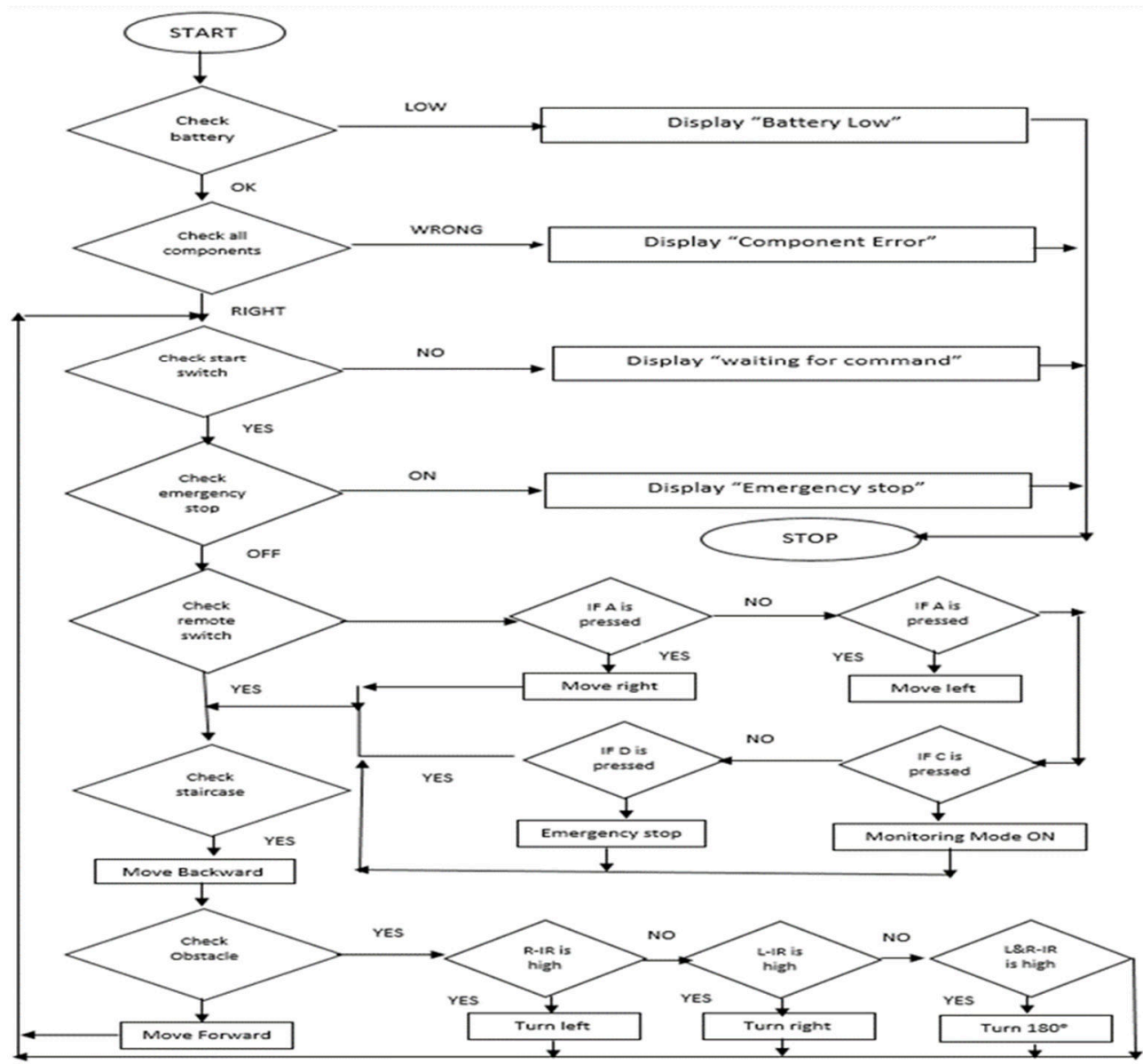

Figure 2. Operational flow chart of the total system. 
When the Bot is on

a. The module checks the power and the working condition of all the components Figure 2 and 3 a.

b. In auto condition after the first step is over the bot starts cleaning the floor in a zigzag manner as shown in Figure 3b.

c. In manual condition, the bot cleans the floor in a spiral path as shown in Figure 3c.

d. Apart from cleaning when the bot is in on condition, it senses the presence of human inside the room and controls the switches of the room.

e. At night mode while there is a random movement of a human, buzzer sounds came for precaution from theft.

f. To avoid any type of accident, there is an emergency stop when the bot reaches staircase, or anybody picks it up.

g. To restrict the Bot inside premises a black strip may be given.

The rough sketch of the bot is shown in Figure $4 \mathrm{a}$ and $\mathrm{b}$. Top view and bottom view of the models are showing the detail components Figure $4 \mathrm{c}$ and $\mathrm{d}$. Our Bot is small in size as shown in Figure $4 \mathrm{e}$, and user-friendly. We have tested the Bot in different environmental conditions with all possible commands and found it suitable as an automatic home appliance.

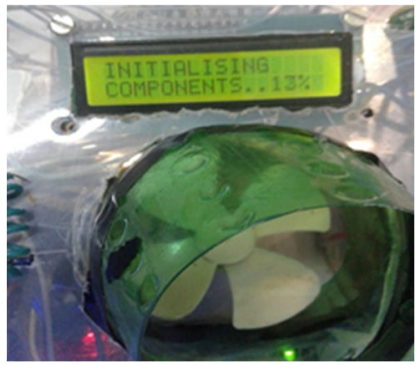

(a)

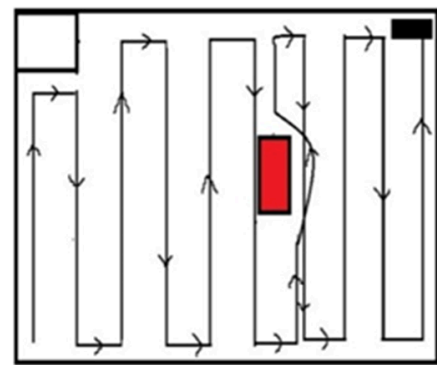

(b)

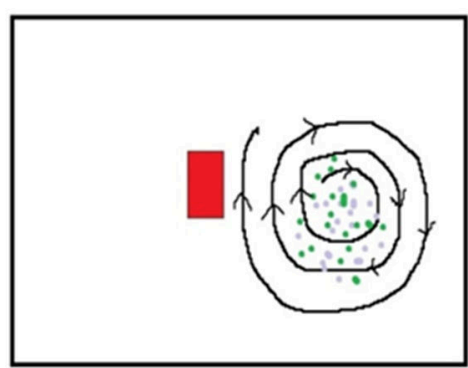

(c)

Figure 3. (a) Initialization of components, (b) zigzag path, (c) Spiral path for floor cleaning

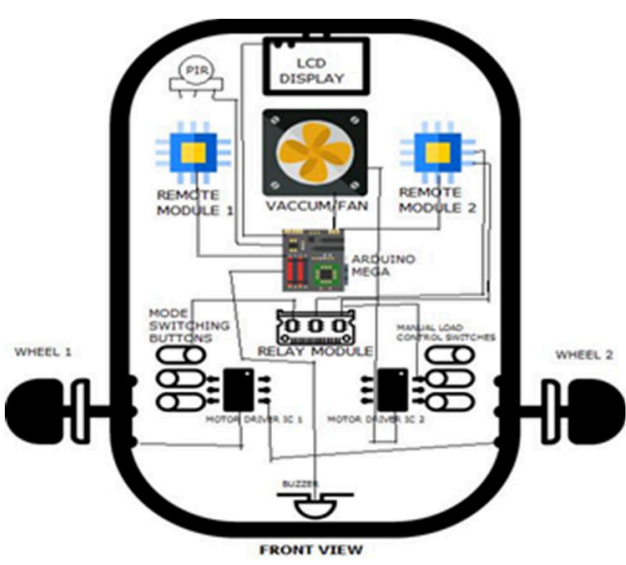

(a)

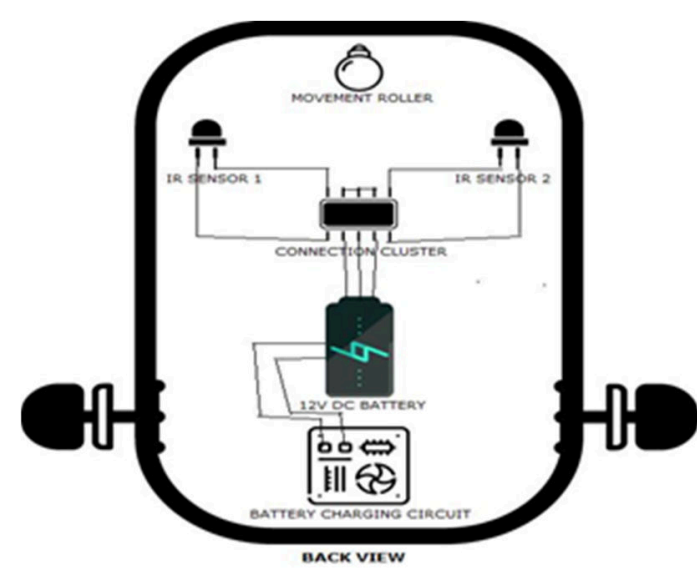

(b) 


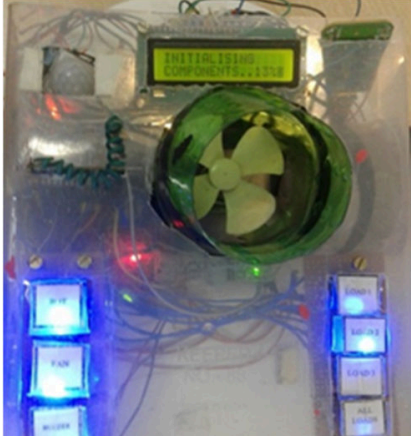

(c)

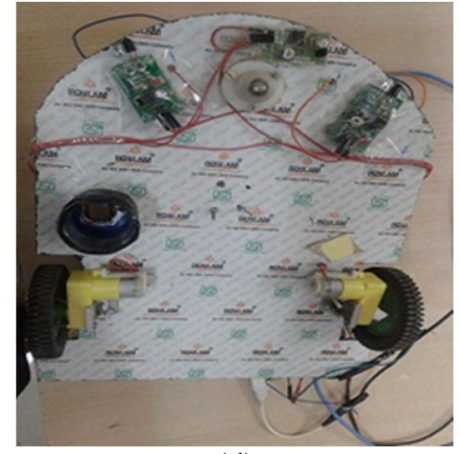

(d)

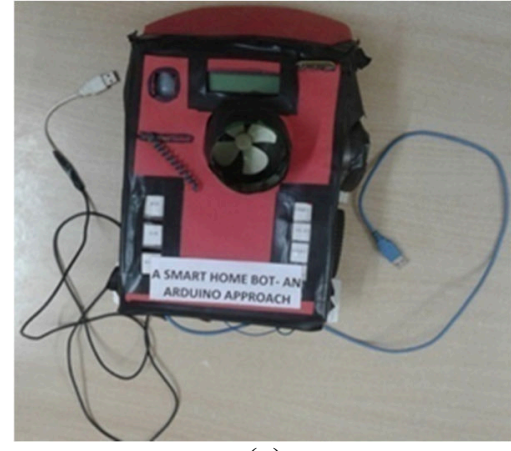

(e)

Figure 4. (a) Top view, (b) a bottom view ofrough sketch, (c) Top view of the model, (d) a bottom view of model, (e) home bot

\section{Conclusion}

The design of a robot for floor cleaning can be used to clean any kind of unreachable places inside the home. The motor used for driving and vacuum cleaner to satisfy the above purpose consumes less power. Micro-controller-based operation for cleaning and controlling reduces the complexity of the overall system. The initial auto troubleshooting increases the time consumption with the cost of the reliability of the system. The operation such as sweeping, controlling, detecting can be performed automatically on any type of floor. Still, new ideas can be implemented in the system to improve its stability and performance in different environmental conditions and geographical area. The research can be directed towards auto detection and cleaning of dust by using image processing. Internet of things (IoT) can be applied for controlling the system remotely.

\section{References}

[1] J Gutmann, K Culp, ME Munich, P Pirjanian. The social impact of a systematic floor cleaner. IEEE Workshop on Advanced Robotics and its Social Impacts (ARSO). 2012: 50-53.

[2] JY Sung, RE Grinter, HI Christensen. Domestic robot ecology. Int. Journal of Social Robotics. 2010; 2(4): 417429.

[3] G Dai, T Chen. Design on Measurement and Control System of Cleaning Robot Based on Sensor Array detection. IEEE International Conference on Control and Automation. 2007: 1319-1322.

[4] Yunbo Hong, Rongchuan Sun, Rui Lin, Shumei Yu, Lining Sun. Mopping module design and experiments of a multifunction floor cleaning robot. Proceeding of the 11th World Congress on Intelligent Control and Automation. 2014: 5097-5102.

[5] SYT Lang, Bing Yung Chee. Coordination of behaviours for mobile robot floor cleaning. Proceedings. 1998 IEEE/RSJ International Conference on Intelligent Robots and Systems. Innovations in Theory, Practice and Applications (Cat. No.98CH36190). 1998; 2:1236-1241.

[6] V Rewatkar, TB Sachin. A Review on Design of Automated Floor Cleaning System. International Journal on Recent and Innovation Trends in Computing and communication. 2015; 2(3): 120-122.

[7] A Thomas, MS Rohith, F Jolly, J Cheriyan, RM George. An Advanced Mobile Robot for Floor Cleaning. National Conference on Recent Advances in Electrical \& Electronics Engineering (NCREEE'16). 2016; 3(5): 102-108.

[8] H Rashid, A Mahmood, S Shekha, SMT Reza, M Rasheduzzaman. Design and development of a DTMF controlled room cleaner robot with two path-following method. 2016 19th International Conference on Computer and Information Technology (ICCIT). 2016: 484-489.

[9] N Prashar, T Thorat, A Galande, R Durande. Cleaning Robot. Global Journal of Computer Science and Technology. 2012; 12(3): 19-22.

[10] J Saunders, DS Syrdal, KL Koay, N Burke, K Dautenhahn. Teach Me-Show Me-End-User Personalization of a Smart Home and Companion Robot. in IEEE Transactions on Human-Machine Systems. 2016; 46(1): 27-40.

[11] A Pandey, A Kaushik, AK Jha, G Kapse.A Technological Survey on Autonomous Home Cleaning Robots. International Journal of Scientific and Research Publications (IJSRP). 2014; 4(4): 1-7.

[12] M Matijevic, V Cvjetkovic. Overview of architectures with Arduino boards as building blocks for data acquisition and control systems. 2016 13th International Conference on Remote Engineering and Virtual Instrumentation (REV). 2016: 56-63. 